\section{Qualitative analysis of microbiologic changes in subgingival biofilm in early stage of fixed orthodontic treatment}

DOI: 10.1515/rrlm-2016-0009

\section{To the Editor:}

Currently, fixed orthodontic therapy is the most frequently used method to treat malocclusions. In addition to its benefits, which are teeth alignment, improvement of occlusion and functional jaw relationship, orthodontic therapy also presents risks and complications. The most common adverse effects of fixed orthodontic appliances are increased supra- and subgingival plaque retention, gingival bleeding and probing depth (1). Brackets or tubes are bonded on the labial surface of permanent teeth, which make oral hygiene and plaque removal difficult. This is one of the reasons why gingivitis, the most frequent side effect of direct bonding, appears in the initial stage of fixed orthodontic treatment.

As soon as orthodontic treatment is applied, the maintenance of a good oral hygiene is crucial and must be thoroughly monitored by the orthodontist in order to minimize plaque accumulation. If this is neglected gingivitis and enamel demineralization (white spot lesions, caries) may develop around and near orthodontic attachments (2).

The periodontal response to bonded orthodontic attachments induced by plaque accumulation can be explained by the alteration of the subgingival microbial composition (3), even when the biofilm composition is altered with aging. A shift in the composition and type of bacteria (4) and elevation of microbial counts in saliva and biofilm can be expected (5). This qualitative bacterial shift seems to be characterized by the growth of anaerobic bacteria accumulated in the subgingival biofilm (6).

DNA-strip technology is a highly sensitive specific PCR technique which allows the isolation, amplification and detection of DNA or RNA from the specimen based on hybridization and alkaline phosphatase reaction on a membrane strip. As a reliable and cost-effective technique, has rapidly become one of the most commonly used techniques in periodontal molecular biology because it is simple, relatively inexpensive and fast. Amplification of DNA fragments is possible from poor quality or minimal quantity of source DNA material (7).

The micro-Ident ${ }^{\circledR}$ plus 11 (Hain Lifescience Gmbh, Germany) test is a qualitative in vitro test for the combined identification of eleven periodontopathogenic bacterial species from subgingival plaque sample. It can detect the following species: Aggregatibacter actinomycetemcomitans (formerly Actinobacillus actinomycetemcomitans), Campylobacter rectus, Capnocytophaga spp. (C. gingivalis, C. ochracea, and C. sputigena). Eikenella corrodens, Eubacterium nodatum, Fusobacterium nucleatum/periodonticum, Parvimonas micra (formerly Peptostreptococcus micros, Micromonas micros), Porphyromonas gingivalis, Prevotella intermedia, Tanerella forsythia (formerly Bacteroides forshytus), Treponema denticola. DNA strips are coated with highly specific probes, which are complementary to selectively amplified nucleic acid sequences.

The aim of our microbiological study was to determine the effect of fixed orthodontic attachments on subgingival biofilm and to evaluate changes that occur in the subgingival microbiota after 4-7 weeks of attachment bonding with use of DNA strip technique. 
We selected nineteen patients (10 females and 9 males) out of those referred for orthodontic treatment to the Orthodontic Department of the University of Medicine and Pharmacy from Tîrgu Mureş, Romania. The mean age of our subjects was $14.6 \pm 1.7$ years. A written informed consent was obtained from each patient or their parents/legal representative before the first sampling. The protocol was reviewed and approved by the Ethical Committee of Scientific Research of the University of Medicine and Pharmacy from Tîrgu Mureş, decision nr. 117/21.11.2013.

The subjects were selected according to the following criteria: (1) good general health, (2) no antibiotic therapy for three months before the initiation of the study and (3) clinically healthy periodontium of first permanent molars with periodontal probing depth less than $3 \mathrm{~mm}$, gingival and plaque index less than 2 at both time points, (4) each patient was applied the same bracket system (Roth Omni, GAC), (5) banding and bonding was performed by using the same light-cured bonding material (Transbond ${ }^{\mathrm{TM}} \mathrm{XT}$, 3M Unitek).

Microbiological subgingival samples were taken from the first permanent molars before (T1) and 4-7 weeks after (T2) the fixed orthodontic attachments were bonded, during the first follow-up. Professional cleaning was not used either before $\mathrm{T} 1$ or before $\mathrm{T} 2$ sampling, in order to maintain the subgingival biofilm intact. The only instructions received by the patients referred to the correct use of manual toothbrush and interdental brush; mouthwash was not prescribed. The molecular-genetic testing of the collected samples was performed by the Department of Microbiology of the University of Medicine and Pharmacy from Tîrgu Mureş. The previously mentioned DNA-strip procedure was divided into three steps: (1) DNA isolation from subgingival samples (QIAamp® DNA Mini Kit, Qiagen, Germany), (2) amplification with two separate amplification reactions using the opti- mized polymerase and primers included in amplification mixes, and (3) manual hybridization.

Prior to sampling, all sites were isolated with cotton rolls and were air dried. The supragingival biofilm was removed with a sterile probe and subgingival plaque was collected by using sterile paper points (Micro-Ident Sampling Set, Hain Lifescience $\mathrm{GmbH}$, Germany) for 30 seconds in five points for each first permanent molar: disto-oral, disto-labial, mesio-oral, mesio-labial and centro-labial points. The paper points were immediately removed from the periodontal sulcus and placed into a screw cap transport tube and DNA isolation was performed. Two separate amplification reactions were used for each sampling optimized polymerase and primers included in the Amplification Mixes A1 (AM-A1 for DNA from Aggregatibacter actinomycetemcomitans, $P$. gingivalis, $P$. intermedia, T. forsythia and $T$. denticola), A2 (AM-A2 for DNA from C. rectus, Capnocytophaga spp, E. corrodens, E. nodatum, F. nucleatum/periodonticum and P. micra) and B (AM-B). Manual hybridization was performed as the last step of the selected procedure. After the strips were completely dried, they were evaluated and interpreted by using the evaluation sheet provided with the kit. In case the test was performed correctly, the control amplifications $\mathrm{AC} 1$ and $\mathrm{AC} 2$ bound to the respective amplification control. We considered valid negative results those in which only the $\mathrm{CC}, \mathrm{HC}, \mathrm{AC} 1$ and AC2 bands were developed.

The Fisher's exact test was used to compare the frequency of the studied periodontopathogens between the two examination times. Statistical analysis was performed using dedicated statistical software (GraphPad InStat) at a significance level of $\mathrm{p}<0.05$.

Results are presented as the percentage of sites positive for each species. The frequency of sites positive at $\mathrm{T} 1$ and $\mathrm{T} 2$ examination times and $p$ values for each microorganism are summarized in Table 1. 
The only putative periodontopathogen bacteria we did not find in any of the T1 samples were the Eubacterium nodatum. Fusobacterium nucleatum was present in the $94.73 \%$ of the T1 samples; Eikenella corrodens and the Capnocytophaga spp. were present in around one-third of the T1 samples. Treponema denticola, Taneralla forsythia, Campylobacter rectus in one-fourth of T1 samples, other bacteria were found randomly.

The frequency of Aggregatibacter actinomycetemcomitans, Parvimonas micra, Porphy- eleven putative periodontopathogens at $\mathrm{T} 1$ was $34.44 \%$ and at T2 was $44.97 \%$, a statistically significant difference was noted between the two sampling times $(\mathrm{p}<0.05)$

Cell culturing, immunological, enzymatic, conventional PCR, real-time PCR and loop-mediated isothermal amplification (LAMP) are the most common microbiological examination methods used in dental practice. Both enzymatic and PCR-based methods are qualitative ones. Even though they use chromosomal DNA and

Tabel 1. Frequency of periodontopathogens in subgingival biofilm at two times of orthodontic treatment: before bonding (T1) and 4-7 weeks after bracket placement (T2)

\begin{tabular}{|c|c|c|c|c|c|}
\hline \multirow[t]{3}{*}{ Periodontopathogen } & \multicolumn{4}{|c|}{ subjects $(n=19)$} & \multirow{3}{*}{$\begin{array}{c}\mathrm{p} \text { value } \\
\mathrm{T} 2 \mathrm{vs} \mathrm{T} 1\end{array}$} \\
\hline & \multicolumn{2}{|c|}{$\mathrm{T} 1$} & \multicolumn{2}{|c|}{$\mathrm{T} 2$} & \\
\hline & $\mathrm{n}$ & $\%$ & $\mathrm{n}$ & $\%$ & \\
\hline Aggregatibacter actinomycetemcomitans (A.a.) & 2 & 10.52 & 2 & 10.52 & 1.00 \\
\hline Porphyromonas gingivalis (P.g.) & 2 & 10.52 & 2 & 10.52 & 1.00 \\
\hline Prevotella intermedia (P.i.) & 2 & 10.52 & 2 & 10.52 & 1.00 \\
\hline Tanerella forsythia (T.f.) & 5 & 26.31 & 8 & 42.10 & .33 \\
\hline Treponema denticola (T.d.) & 6 & 31.57 & 9 & 47.36 & .50 \\
\hline Parvimonas micra (P.m.) & 3 & 47.36 & 9 & 47.36 & 1.00 \\
\hline Fusobacterium nucleatum (F.n.) & 18 & 94.73 & 19 & 100 & .31 \\
\hline Campylobacter rectus (C.r.) & 5 & 26.31 & 8 & 42.10 & .078 \\
\hline Eubacterium nodatum (E.n.) & 0 & 0 & 2 & 10.52 & .375 \\
\hline Eikenella corrodens (E.c.) & 14 & 73.68 & 16 & 84.21 & .313 \\
\hline Capnocytophaga spp. (C.sp.) & 15 & 78.94 & 17 & 89.47 & .301 \\
\hline Overall frequency of positive sites & & 34.44 & & 44.97 & $.035^{*}$ \\
\hline
\end{tabular}

a Fisher's exact test; * $\mathrm{P}<.05$.

romonas gingivalis and Prevotella intermedia species did not change at T2 compared with T1. Campylobacter rectus, Capnocytophaga spp., Eikenella corrodens, Eubacterium nodatum, Fusobacterium nucleatum/periodonticum, Tanerella forsythia and Treponema denticola species showed moderate elevation of frequency at T2 compared with T1 examination time. No statistically significant differences in frequency were observed for the above mentioned seven investigated species between the two sampling times.

The frequency of sites positive for all the



Figure 1. Comparison of detection frequency of the 11 periodontopathogens between the two examination times. 
they cannot discriminate viable bacteria from dead ones, PCR-based methods are relatively sensitive and the use of species-specific primers allows the identification of the presence of specific bacteria without any information of their amount (8). Our aim was to evaluate the qualitative change of subgingival microbiota of a certain locus - the gingival sulcus of the first permanent molars - right after orthodontic attachments were placed, using a DNA amplification method.

Some clinical studies revealed more severe periodontal complications in the distal part of the dental arches, which can be explained by the presence of molar bands and poor oral hygiene in this region (9). Usually fixed orthodontic therapy starts with the anchorage of teeth by bonding or banding, this is the reason why we have chosen to evaluate the subgingival biofilm of the first permanent molars.

Bacterial species involved in the initiation and progression of periodontal disease have been classified, divided into groups labeled with colours (10). The different categories were classified according to the pathogenity of the bacteria and their role in the development of the periodontal responses of the subgingival biofilm. The red complex contained Porphyromonas gingivalis, Tannerella forsythia and Treponema denticola, bacteria that can be associated with the severe forms of some periodontal diseases. The orange complex comprised Campylobacter gracilis, Campylobacter rectus, Campylobacter showae, Eubacterium nodatum, Fusobacterium nucleatum, Peptostreptococcus micros, Prevotella intermedia, Prevotella nigrescens, Streptococcus constellatus. Some studies revealed that in the subgingival biofilm of deep pockets from adults with periodontitis, the presence of $P$. intermedia can always be associated with $F$. nucleatum $(3,6)$. The yellow complex comprised Streptococcus gordonii,
Streptococcus intermedius, Streptococcus mitis, Streptococcus oralis and Streptococcus sanguis.

Periodontopathogen species investigated in this study like Porphyromonas gingivalis, Tanerella forsythia and Treponema denticola are called "red complex" species and they are associated with adult periodontitis (10), along with other virulent species such as Eubacterium nodatum, Fusobacterium nucleatum/periodonticum and gingivitis associated species, like Eikenella corrodens and Campylobacter rectus.

The frequency of positive sites significantly increased $(p=0.035)$ from $\mathrm{T} 1$ to $\mathrm{T} 2$ sampling time. This result confirms studies that concluded that a fixed orthodontic appliances may affect the composition of subgingival microbiota, increasing the prevalence of periodontopathogens $(11,12)$.

A slight increase in frequency for Campylobacter rectus, Capnocytophaga spp, Eikenella corrodens, Eubacterium nodatum, Fusobacterium nucleatum/periodonticum, Tanerella forsythia and Treponema denticola was observed between the two examination times, but no significant differences were observed. Other similar studies reported a high frequency for the above mentioned species before appliance removal and a significant decrease of their frequency after debonding on subjects with signs of gingival inflammation $(13,14)$.

Eubacterium nodatum was the only one missing bacteria from $\mathrm{T} 1$ samples. The most common bacteria in both groups were Fusobacterium nucleatum, followed by Capnocytophaga spp. and Eikenella corrodens. Fusobacterium nucleatum is known as a second colonizer and the first Gram-negative species established in plaque biofilms (15).

Studying the mechanism of subgingival biofilm formation, it has become obvious that there is a certain succession of bacterial colonization in the gingival crevicular fluid when periodon- 
tal inflammation appears. Within a few days after the appearance in subgingival biofilm of early colonizers (Streptococci and Actinomyces species), the development of a complex microbial community can be observed and secondary colonizers tend to be the more pathogenic species such as Aggregatibacter actinomycetemcomitans, Fusobacterium nucleatum, Porphyromonas gingivalis, Tannerella forsythia, Treponema denticola (16). Our results showed that the presence of Aggregatibacter actinomycetemcomitans showed no difference between $\mathrm{T} 1$ and $\mathrm{T} 2$ groups, and the two cases in which it was found showed the most diverse composition of subgingival plaque. Second colonizer Fusobacterium nucleatum was found in the highest percentage in both samples, Tannerella forsyth$i a$, Treponema denticola increased in three cases, and the percentage of Porphyromonas gingivalis did not change. These findings were also reported by several studies $(17,18)$ which concluded, that the placement of an orthodontic appliance affects the subgingival microbial composition in early phases of the orthodontic treatment, the prevalence of putative periodontopathogens increase, especially in the molar region (19).

The frequency of Campylobacter rectus and Eikenella corrodens of $26.31 \%$ and $73.68 \%$ for T1 increased to $42.10 \%$ and $84.21 \%$ at T2 with no statistical significant differences. Several studies found these two species at higher prevalence in gingivitis (20) and suggested that they might be considered as contributors in the development of periodontitis. Other studies found these two species in a relatively high frequency, indicating that they may be part of normal subgingival flora in subjects with healthy gingiva (21).

The major aim of this study was to evaluate whether the initial subgingival biofilm composition changes in early phase of fixed orthodontic treatment. The relatively short time between the two examination times showed that significant differences in overall species frequency can be expected and therefore, it is recommended that all orthodontic patients should receive oral hygiene instruction and professional prophylaxis at the very beginning of the orthodontic treatment.

The number of selected subjects can be considered as a limitation of our study and it is due to the fact that we focused on orthodontic patients with good oral hygiene and no alteration of gingival parameters gingival from $\mathrm{T} 1$ to $\mathrm{T} 2$ examination time. Further subject recruitment and evaluation is needed.

Based on our findings we conclude that overall frequency of the studied eleven periodontopathogens significantly increased in subgingival microbiota in the first 4-7 weeks of orthodontic treatment. Tanerella forsythia, Treponema denticola, Fusobacterium nodatum, Campylobacter rectus, Eubacterium nodatum, Eikenella corrodens and Capnocytophaga spp. frequency slightly increased 4-7 weeks after the fixed orthodontic attachments were bonded. Plaque control in order to maintain gingival health is important before and as soon as orthodontic treatment is applied and additional periodontal prophylactic measures are indicated.

\section{Krisztina Ildiko Mártha ${ }^{1 *}$, Cristina Ioana Bică르, Luminița Lazăr ${ }^{3}$, Réka Gyergyay ${ }^{4}$, Anna Lőrinczi ${ }^{5}$, Alpár Dakó ${ }^{5}$, Lilla Katalin Lőrinczi ${ }^{6}$}

1. Orthodontic Department, Faculty of Dentistry, University of Medicine and Pharmacy Tîrgu Mureș, Romania;

2. Pediatric Dentistry Department, Faculty of Dental Medicine, University of Medicine and Pharmacy Tîrgu Mureș, Romania;

3. Parodontology Department, Faculty of Dentistry, University of Medicine and Pharmacy Tîrgu Mureș, Romania;

4. Pediatric Dentistry Department, Faculty of 
Dentistry, University of Medicine and Pharmacy Tîrgu Mureș, Romania;

5. Faculty of Dentistry, University of Medicine and Pharmacy Tîrgu Mureș, Romania;

6. Microbiology Department, University of Medicine and Pharmacy Tîrgu Mureș, Romania;

*Corresponding author: Krisztina Ildiko Mártha email:marthakriszti@yahoo.com

Received: 22 $2^{\text {th }}$ October 2015; Accepted: $27^{\text {th }}$ February 2016; Published: $12^{\text {th }}$ March 2016

\section{References}

1. Alexander SA. Effects of orthodontic attachments on the gingival health of permanent second molars. Am J Orthod Dentofacial Orthop. 1991;100:337-340. DOI: 10.1016/0889-5406(91)70071-4

2. Derks A, Katsaros C, Frencken JE, van't Hof MA, Kuijpers-Jagtman AM. Caries-inhibiting effect of preventive measures during orthodontic treatment with fixed appliances. A systematic review. Caries Res. 2004 SepOct;38(5):413-20. DOI: 10.1159/000079621

3. Kim S-H, Choi D-S, Jang I, Cha B-K, Jost-Brinkmann $\mathrm{P}-\mathrm{G}$, Song J-S. Microbiologic changes in subgingival plaque before and during the early period of orthodontic treatment. Angle Orthod. 2012;82(2):254-60. DOI: 10.2319/030311-156.1

4. Torlakovic L, Paster BJ, Ogaard B, Olsen I. Changes in the supragingival microbiota surrounding brackets of upper central incisors during orthodontic treatment. Acta Odontol Scand. 2013;71(6):1547-54. DOI: 10.3109/00016357.2013.776107

5. Hakan Türkkahraman, Mözgür Sayın, F. Yeşim Bozkurt, Zuhal Yetkin, Selçuk Kaya, Süleyman Önal. Archwire Ligation Techniques, Microbial Colonization, and Periodontal Status in Orthodontically Treated Patients. Angle Orthod. 2005;75(2):231-6.

6. Garcez AS, Suzuki SS, Ribeiro MS, Mada EY, Freitas AZ, Suzuki H. Biofilm retention by 3 methods of ligation on orthodontic brackets: A microbiologic and optical coherence tomography analysis. Am J Orthod Dentofacial Orthop. $2011 ; 140(4): 193-8$. DOI: $10.1016 / j$. ajodo.2011.04.019

7. Joshi M, Deshpande J.D. Polymerase chain reaction: methods, principles and application. Int J Biomed Res. 2010;1(5):81-97.

8. Shaddox, L.M. \& Walker, C. Microbial testing in periodontics: value, limitations and future directions. Periodontology 2000; 50(1):25-38. DOI: 10.1111/j.16000757.2008.00285.x

9. Corbacho de Melo MM, Cardoso MG, Faber J, Sobral A. Risk factors for periodontal changes in adult pa- tients with banded second molars during orthodontic treatment. Angle Orthod. 2012 Mar;82(2):224-8. DOI: 10.2319/030911-172.1

10. Socransky SS, Haffajee AD, Cugini MA, Smith C, Kent RL. Jr. Microbial complexes in subgingival plaque. J Clin Microbiol. 1998; 25(2):134- 44. DOI: 10.1111/j.1600-051x.1998.tb02419.x

11. Lee SM, Yoo SY, Kim HS, et al. Prevalence of putative periodontopathogens in subgingival dental plaques from gingivitis lesions in Korean orthodontic patients. J Microbiol. 2005;43:260-5.

12. Miethke RR, Bernimoulin JP. Effects of bands and brackets on the marginal periodontium. Fortschr Kieferorthop. 1988; 49:160-169. DOI: 10.1007/ BF02163375

13. Sallum EJ, Nouer DF, Klein MI, Goncalves RB, Machion L, Wilson Sallum A, Sallum EA. Clinical and microbiologic changes after removal of orthodontic appliances. Am J Orthod Dentofacial Orthop. 2004;126:363-6. DOI: 10.1016/j.ajodo.2004.04.017

14. Yang YM, Kim SS, Jun ES, Park SB. Changes of periodontopathogens and clinical parameters of periodontal tissue after debanding. Korean J Orthod. 2006;36:263-74.

15. Benoit Signat, Christine Roques, Pierre Poulet, Danielle Duffaut Fusobacterium nucleatum in periodontal health and disease. Curr Issues Mol Biol. 2011; 13(2):25-36.

16. Kolenbrander PE, Andersen RN, Blehert DS, Egland PG, Foster JS, Palmer RJ. Communication among oral bacteria. Microbiol Mol Biol Rev. 2002;66:486-505. DOI: 10.1128/MMBR.66.3.486-505.2002

17. Kim K, Heimisdottir K, Gebauer U, Persson GR. Clinical and microbiological findings at sites treated with orthodontic fixed appliances in adolescents. Am J Orthod Dentofacial Orthop 2010;137(2):223-8. DOI: 10.1016/j.ajodo.2008.03.027

18. Levrini L, Mangano A, Montanari P, Margherini S, Caprioglio A, Abbate GM. Periodontal health status in patients treated with the Invisalign ${ }^{\circledR}$ system and fixed orthodontic appliances: A 3 months clinical and microbiological evaluation. Eur J Dent. 2015;9(3):40410. DOI: $10.4103 / 1305-7456.163218$

19. Kim SH, Choi DS, Jang I, Cha BK, Jost-Brinkmann PG, Son JS. Microbiologic changes in subgingival plaque before and during the early period of orthodontic treatment. Angle Orthod. 2012; 82(2):254-60. DOI: 10.2319/030311-156.1

20. Ashimoto A, Chen C, Bakker I, Slots J. Polymerase chain reaction detection of 8 putative periodontal pathogens in subgingival plaque of gingivitis and advanced periodontitis lesions. Oral Microbiol Immunol. 1996;11:266-73. DOI: 10.1111/j.1399-302X.1996. tb00180.x

21. Chen CK, Wilson ME. Eikenella corrodens in human oral and non-oral infections: a review. J Periodontol. 1992;63:941-53. DOI: 10.1902/jop.1992.63.12.941 